\title{
Prediction of Persistent Impaired Glucose Tolerance in Patients with Minor Ischemic Stroke or Transient Ischemic Attack
}

\author{
Elizabeth Osei, MD, ${ }^{*}$ Heleen M. den Hertog, MD, PhD, + \\ Susanne Fonville, MD, PhD, $\ddagger$ Paul J.A.M. Brouwers,§ Laus J.M.M. Mulder, $\|$ \\ Peter J. Koudstaal, MD, PhD, $\mid$ Diederik W.J. Dippel, MD, PhD, $\emptyset$ \\ Adrienne A.M. Zandbergen, MD, PhD, $\uparrow$ and Hester F. Lingsma, $\uparrow$
}

\begin{abstract}
Background: Impaired glucose tolerance (IGT) in patients with ischemic stroke can return to normal, reflecting an acute stress response, or persist. Persistent IGT is associated with an increased risk of recurrent stroke, other cardiovascular diseases and unfavorable outcome after stroke. We aim to validate our previously developed model to identify patients at risk of persistent IGT in an independent data set, and, if necessary, update the model. Methods: The validation data set consisted of 239 nondiabetic patients with a minor ischemic stroke or TIA and IGT in the acute phase (2-hour post-load glucose levels between 7.8 and $11.0 \mathrm{mmol} / \mathrm{l}$ ). The outcome was persistent versus normalized IGT, based on repeated oral glucose tolerance test after a median of 46 days. The discriminative ability of the original model was assessed with the area under the ROC curve (AUC). The updated model was internally validated with bootstrap resampling and cross-validated in the development population of the original model. Results: One-hundred eighteen of 239 (49\%) patients had persistent IGT. The original model, with the predictors age, current smoking, statin use, triglyceride, hypertension, history of cardiovascular diseases, body mass index (BMI), fasting plasma glucose performed poorly (AUC .60). The newly developed model included only BMI, hypertension, statin use, atrial fibrillation, 2-hour post-load glucose levels, $\mathrm{HbA1c}$, large artery atherosclerosis, and predicted persistent IGT more accurately (internally validated AUC 0.66, externally validated AUC .71). Conclusions: This prediction model with simple clinical variables can be used to predict persistent IGT in patients with IGT directly after minor stroke or TIA, and may be useful to optimize secondary prevention by early identification of patients with disturbed glucose metabolism.
\end{abstract}

Key Words: Glucose-stroke-TIA-prediction-prediabetes-treatment (c) 2020 Elsevier Inc. All rights reserved.

From the *Flevoziekenhuis, Almere, the Netherland; tIsala, Zwolle, the Netherlands; $¥$ Spaarne Gasthuis, Haarlem, the Netherlands; §Medisch Spectrum Twente, Enschede, the Netherlands; ||Ikazia Ziekenhuis, Rotterdam, the Netherlands; and $₫$ Erasmus Medical Center, Rotterdam, the Netherlands.

Received February 14, 2020; revision received March 7, 2020; accepted March 13, 2020.

Grant Support: None.

Address correspondence to Elizabeth Osei, MD, Flevoziekenhuis, Postbus 3005, 1300EG Almere, the Netherlands. E-mail:

1.osei.neurologie@gmail.com.

$1052-3057 / \$$ - see front matter

(c) 2020 Elsevier Inc. All rights reserved.

https://doi.org/10.1016/j.jstrokecerebrovasdis.2020.104815

\section{Introduction}

Impaired glucose tolerance (IGT) is an intermediate metabolic state between normal glucose metabolism and diabetes mellitus, with a growing prevalence worldwide. IGT is highly prevalent in patients with recent ischemic stroke or transient ischemic attack (TIA). This IGT can be transient, reflecting an acute stress response, or persistent, representing undiagnosed impaired glucose metabolism. $^{1}$ Of nondiabetic stroke patients with IGT in the acute phase after stroke and repeated glucose assessment after 3 months, $22 \%-47 \%$ has persistent IGT after 3 months. $^{1-4}$

IGT increases the risk of recurrent stroke and other cardiovascular events. ${ }^{5,6}$ Moreover, IGT is also associated with poor functional outcome and mortality after stroke., 5,8 
Glucose lowering medication or lifestyle interventions may be beneficial for these patients, in the acute phase as well as part of secondary prevention. In a previous study by our group, we developed a prediction model to identify patients with persistent impaired glucose tolerance at 3 months after TIA and ischemic stroke. This prediction model including age, current smoking, statin use, triglyceride, hypertension, previous ischemic cardiovascular disease, body mass index, and fasting plasma glucose accurately predicted persistent IGT. ${ }^{4}$ However, this prediction model has not been externally validated in an independent population.

The aim of our study was to externally validate the original prediction model and, if necessary, to update the model to identify patients at risk of persistent IGT after TIA and minor stroke.

\section{Methods}

\section{Study Population}

Patients were included if they were 18 years or older and attended the TIA outpatient clinic, or were admitted to the stroke unit of Medical Spectrum Twente in Enschede and Ikazia Hospital in Rotterdam, the Netherlands between February 2014 and December 2017. These patients had a clinical diagnosis of TIA, amaurosis fugax or minor ischemic stroke (defined as a modified Rankin scale score of 3 or less) within the previous 6 months. Written informed consent was obtained from all patients or a first-degree relative, as approved by the Institutional Ethics Committee.

\section{Patient Characteristics}

Demographic data, vascular history and risk factors, including statin use, hypertension (defined as previous use of antihypertensive medication or blood pressure higher than 140/90 mmHg), current smoking, atrial fibrillation, body mass index (BMI), laboratory assessments, including lipid profile and ischemic stroke subtype according to the TOAST classification ${ }^{9}$ were collected.

\section{Glucose Assessment}

In all nondiabetic patients (defined as no use of oral or parenteral antidiabetic medication), fasting plasma glucose and post-load glucose levels were assessed on the day of the TIA outpatient clinic visit or the second day of admission on the stroke unit as part of standard care. Post-load glucose levels were assessed by performing the oral glucose tolerance test (OGTT). The OGTT was performed after overnight fasting, and patients drank a solution of $75 \mathrm{~g}$ glucose in $150 \mathrm{~mL}$ water. Eligible patients were diagnosed with IGT, defined as 2-hour post-load glucose levels between 7.8 and $11.0 \mathrm{mmol} / \mathrm{L}^{10}$ The glycosylated hemoglobin A1c levels ( $\mathrm{HbA} 1 \mathrm{c})$ were assessed at the follow-up visit, 2 to 12 weeks after admission and at 6 months.

\section{Outcome Measures}

Two to 12 weeks after the initial OGTT, patients with IGT were asked to undergo a second OGTT. We previously stated in the study protocol that the OGTT should be repeated after 2-6 weeks. Due to logistic reasons, this time frame was extended to 2-12 weeks. Patients with fasting plasma glucose levels of $7.0 \mathrm{mmol} / \mathrm{L}$ or higher were diagnosed with diabetes and therefore did not undergo the repeated OGTT.

Based on the results of the second OGTT, patients were classified in the following 2 groups: normalized post-load-glucose levels (2-hour post-load-glucose levels $<7.8 \mathrm{mmol} / \mathrm{L}$ ) or persistent IGT (2-hour post-load glucose levels $\geq 7.8 \mathrm{mmol} / \mathrm{L}$ according to international guidelines ([10]).

\section{Statistical Analysis}

We used means with standard deviations to describe normally distributed continuous variables, and medians and interquartile ranges to describe the non-normally distributed continuous variables. We compared patient characteristics between glucose groups, with normalized postload-glucose levels as a reference. The differences between categorical variables were tested by chi-square and continuous variables by Student's t-test. Non-normally distributed variables were compared by Mann-Whitney Utest. $P<.05$ was considered to indicate statistical significance. Missing data were imputed with single imputation using baseline characteristics and the outcome variable, if the frequency was less than $5 \%$. The analysis was carried out with STATA 12.1 statistical package (Statacorp, College Station, Texas) and R statistical software.

\section{Model Validation}

First, we compared patient characteristics between the current study and the previous (development cohort $)^{4}$ Second, to assess the validity of the original prediction model, we assessed the effect of the predictors of the original model in the current development population. As the final validation step, overall model performance was assessed in terms of discrimination and calibration. Calibration refers to the agreement between observed and predicted outcomes. Calibration plots were used to visualize the observed and predicted rates. The intercept shows whether predictions are systematically too low or too high, and should ideally be zero. The calibration slope reflects the average effects of the predictors in the model, and was estimated in a logistic regression model. In a perfect model, the slope is 1 .

Discrimination refers to the ability to distinguish a patient with and without persistent IGT, with the use of the area under the ROC curve (AUC). The AUC ranges from .5 for noninformative to 1.0 for perfect models. ${ }^{11}$ 


\section{Model Development}

The next, optional third step was to develop a new, updated model. Possible predictors of persistent IGT included known risk factors for developing diabetes and other vascular risk factors according to the previous literature: age, sex, current smoking, statin use, hypertension, previous ischemic cardiovascular disease, atrial fibrillation, BMI, TIA versus ischemic stroke, large artery atherosclerosis, fasting plasma glucose, 2-hour post-load glucose, and $\mathrm{HbA1c}$ levels. ${ }^{1-3,12-15}$ Large artery atherosclerosis was defined as significant $(>50 \%)$ stenosis or occlusion of a major brain artery or branch cortical artery, due to atherosclerosis. ${ }^{9}$

All potential predictors were tested with a multivariable logistic regression model and tested for the final model with stepwise backward selection, with a $P$-value of less than or equal to .2. The internal validity of the model was assessed by means of bootstrapping techniques, resulting in an internally validated AUC. A bootstrap sample means that a random sample of the same size of the original population is drawn with replacement. The model is developed in the bootstrap sample and evaluated in the original sample. To obtain stable results, the procedure has to be repeated multiple times.For correction of too optimistic estimation of the predictive value, we performed shrinkage of the regression coefficients based on the difference between the apparent and internally validated AUC.

As a fourth step, we assessed the external validity of the new updated model by testing the performance of the new model in the population that was used to develop the original model. ${ }^{4}$

\section{Results}

\section{Study Population}

A total of 239 patients had IGT based on the first OGTT. Mean age was 68 years (SD 10 years), $134(56 \%)$ patients were men, $136(57 \%)$ had ischemic stroke, 158 (66\%) had hypertension, and 195 (82\%) used statins.

Of the total of 239 patients, $118(49 \%)$ had persistent IGT based on the repeated OGTT performed after a median of 46 days (IQR 33). In patients with persistent IGT, mean BMI, $\mathrm{HbA1c}$, triglycerides and LDL-levels were significantly higher than patients with normalized IGT. Patients with persistent IGT more frequently had hypertension and atrial fibrillation than patients with normalized IGT. (Table 1)

A total of 105 patients were included within 6 weeks after TIA or ischemic stroke and 11 patients around 6 months after the event. The percentage of persistent IGT was quite similar in both groups: 52 of the 105 patients $(50 \%)$ and 6 of the 11 patients (55\%) respectively.

\section{External Validation of Original Model}

Compared to the patient characteristics of the previous original model, ${ }^{4}$ in our present study mean age was higher, current smoking was less frequent, whereas hypertension, statin use, TIA and large artery atherosclerosis, and small vessel disease were more frequent. Comparing the characteristics between patients with transient and those with persistent IGT, we found a significant difference in percentage of patients with atrial fibrillation, mean BMI and 2-hour post-load glucose levels, which was not the case in the previous study. ${ }^{4}$ In addition, in our present study there was no significant difference in percentage of statin use and in mean age between patients with or without persistent IGT, contrary to the previous study.

Overall, the performance of the original model was poor with an AUC of .60, calibration slope of .22 and intercept of -2.97 (Fig 1). ${ }^{11}$ Therefore, we developed a new, updated model.

\section{Model Development}

The following predictors from the multivariable logistic regression were selected for the new prediction model after stepwise backward selection: BMI, hypertension, statin use, atrial fibrillation, large artery atherosclerosis, first 2-hour post-load glucose levels, and $\mathrm{HbA1c}$ levels. (Table 2) The AUC of the newly developed model was 0.72 and the internally validated bootstrapped AUC .66. For correction of too optimistic estimation of the predictive value, shrinkage of the regression coefficients was performed (Table 3).

The risk of persistent IGT in patients with ischemic stroke or TIA can be calculated as follows: exp(linear predictor)/(1 $+\exp [$ linear predictor] $)$. Linear predictor $=-7.4706+.0529 \times$ $\mathrm{BMI}+.3924 \times$ hypertension $+.3668 \times$ statin use $+.8444 \times$ atrial fibrillation $+.2598 \times 2$-hour post-load glucose levels $+.0793 \times \mathrm{Hba} 1 \mathrm{c}+.3579 \times$ large artery atherosclerosis.

The model was externally validated in the population of the previous original model, which showed the discriminative ability (AUC) of .71, calibration slope of 1.15 and intercept of .3 (Fig 2).

\section{Discussion}

In our study, 118 of the 239 patients (49\%) with IGT after a minor ischemic stroke or TIA had persistent IGT in the 2 to 12 weeks after initial assessment. Our original prediction model did not perform well in our new dataset. Therefore, we developed a new updated prediction model that more accurately predicts persistent IGT in patients with minor ischemic stroke or TIA. The predictors were BMI, hypertension, statin use, atrial fibrillation, large artery atherosclerosis, 2-hour post-load glucose levels and $\mathrm{HbA1c}$ levels. The prediction model still performed well when we externally validated it in the population of our previous study, ${ }^{4}$ which had a comparable proportion of patients with persistent IGT of $47 \%$.

Age, current smoking, triglycerides and fasting plasma glucose were not predictors in our study, in contrast to our previous study. ${ }^{4}$ Possible reasons are that increasing age 
Table 1. Patient characteristics compared between transient impaired glucose tolerance and persistent impaired glucose tolerance

\begin{tabular}{|c|c|c|c|c|}
\hline & $\begin{array}{l}\text { Total population } \\
(\mathrm{n}=239)\end{array}$ & $\begin{array}{l}\text { Transient impaired } \\
\text { glucose tolerance } \\
(\mathrm{n}=121)\end{array}$ & $\begin{array}{l}\text { Persistent impaired } \\
\text { glucose tolerance } \\
(\mathrm{n}=118)\end{array}$ & $P$ value \\
\hline Age, years (SD) & $68(10)$ & $68(10)$ & $69(11)$ & .30 \\
\hline Sex (male), n (\%) & $134(56 \%)$ & $70(58 \%)$ & $64(54 \%)$ & .57 \\
\hline \multicolumn{5}{|l|}{ Vascular risk factors } \\
\hline Current smoking, n (\%) & $35(15 \%)$ & $16(13 \%)$ & $19(16 \%)$ & .53 \\
\hline $\mathrm{BMI}$ in $\mathrm{kg} / \mathrm{m}^{2}$, mean $(\mathrm{SD})$ & $27(4)$ & $26(4)$ & $28(4)$ & $<.01$ \\
\hline Hypertension, $\mathrm{n}(\%)$ & $158(66 \%)$ & $71(59 \%)$ & $87(74 \%)$ & .01 \\
\hline Statin use, $\mathrm{n}(\%)$ & $195(82 \%)$ & $94(78 \%)$ & $101(86)$ & .12 \\
\hline Atrial fibrillation, $\mathrm{n}(\%)$ & $24(10 \%)$ & $6(5 \%)$ & $18(15 \%)$ & $<.01$ \\
\hline \multicolumn{5}{|l|}{ Vascular history } \\
\hline Ischemic cardiovascular disease, $\mathrm{n}(\%)$ & $72(30 \%)$ & $37(31 \%)$ & $35(30 \%)$ & .88 \\
\hline \multicolumn{5}{|l|}{ Event } \\
\hline Ischemic stroke, $\mathrm{n}(\%)$ & $136(57 \%)$ & $68(56 \%)$ & $68(58 \%)$ & .82 \\
\hline TOAST classification & & & & .09 \\
\hline Large artery atherosclerosis, $\mathrm{n}(\%)$ & $33(14 \%)$ & $14(12 \%)$ & $19(16 \%)$ & \\
\hline Cardioembolism, $\mathrm{n}(\%)$ & $26(11 \%)$ & $8(7 \%)$ & $18(15 \%)$ & \\
\hline Small vessel occlusion, $\mathrm{n}(\%)$ & $117(49 \%)$ & $61(50 \%)$ & $56(48 \%)$ & \\
\hline Other determined etiology, $\mathrm{n}(\%)$ & $2(1 \%)$ & $1(1 \%)$ & $1(1 \%)$ & \\
\hline Unknown, $\mathrm{n}(\%)$ & $60(25 \%)$ & $37(31 \%)$ & $23(20 \%)$ & \\
\hline \multicolumn{5}{|l|}{$\begin{array}{l}\text { Glucose assessment during admission/ } \\
\text { visiting TIA clinic }\end{array}$} \\
\hline $\begin{array}{l}\text { Fasting glucose levels, mean (SD), } \\
\text { mmol/L }\end{array}$ & $5.5(.5)$ & $5.5(.5)$ & $5.5(.8)$ & .35 \\
\hline $\begin{array}{l}\text { 2-hour postload glucose levels, mean } \\
\text { (SD), mmol/L }\end{array}$ & $9.1(1)$ & $8.9(.8)$ & 9.4.9) & $<.01$ \\
\hline HbAlc, mean (SD), mmol/mol & $38(3)$ & $37(3.3)$ & $38(3.1)$ & $<.01$ \\
\hline $\begin{array}{l}\text { Days between event and first OGTT, } \\
\text { median (IQR) }\end{array}$ & $46(33)$ & $44(30)$ & $47(36)$ & .69 \\
\hline \multicolumn{5}{|l|}{ Lipid profile } \\
\hline Total cholesterol, mean (SD), mmol/L & $5(1.2)$ & $5.0(1.2)$ & $4.9(1.1)$ & .35 \\
\hline Triglycerides, mean (SD), mmol/L & $1.5(.7)$ & $1.3(.7)$ & $1.4(.7)$ & .05 \\
\hline HDL-cholesterol, mean (SD), mmol/L & $2.9(1)$ & $1.7(.8)$ & $1.4(.6)$ & $<.01$ \\
\hline LDL-cholesterol, mean (SD), mmol/L & $1.3(.6)$ & $2.9(1)$ & $3(.9)$ & .58 \\
\hline
\end{tabular}

Abbreviations: BMI, body mass index; HbA1c, glycolysated Haemoglobin 1c; IQR, interquartile range; OGTT, oral glucose tolerance test; SD, standard deviation; TIA, transient ischemic attack; TOAST, Trial of Org 10172 in Acute Stroke Treatment.

and currently smoking are variables that are generally associated with cardiovascular diseases, but perhaps not specific enough to predict IGT. Also, triglycerides in this range are less associated with cardiovascular diseases than LDLcholesterol. ${ }^{16}$ In addition, 2-hour post-load glucose levels and $\mathrm{HbA} 1 \mathrm{c}$ levels may be a better reflection of disturbed glucose metabolism than fasting glucose levels alone. ${ }^{17}$ However, diabetes is less frequently identified with Hba1c levels compared with fasting glucose levels and 2-hour post-load glucose levels. ${ }^{10}$ In addition, in our study population of stroke patients, 2-hour post-load glucose levels and $\mathrm{HbA1c}$ levels may be a better reflection of disturbed glucose metabolism than fasting glucose levels alone.

In concordance with previous studies that tried to predict persistent IGT, 2-hour post-load glucose levels were a significant predictor. ${ }^{1-3}$ Also, the proportion of persistent IGT in these studies was comparable with our study and varied between $41 \%$ - $69 \%$. A difference was that in our previous original study and the other previous mentioned studies, the OGTT was repeated at 3 months, which is later than the median of approximately 6 weeks in our current study. Also, the predictors of persistent IGT were different among the studies which shows that persistent IGT is difficult to predict. Lifestyle modification after TIA or ischemic stroke could also improve glucose metabolism, ${ }^{18}$ which also influences the prediction of persistent IGT.

To date, we performed the only study which externally validated a prediction model to predict persistent IGT. Our study provides evidence that IGT in patients with ischemic stroke or TIA is not only due to a stress reaction, but in half of the patients can be due to underlying abnormal glucose metabolism. It is important to predict which patients have persistent IGT. IGT increases the risk of developing type 2 diabetes. ${ }^{19,20}$ IGT can also increase the risk of recurrent stroke and other cardiovascular diseases ${ }^{5,21}$ and is associated with poor outcome after stroke. ${ }^{8,22}$ 


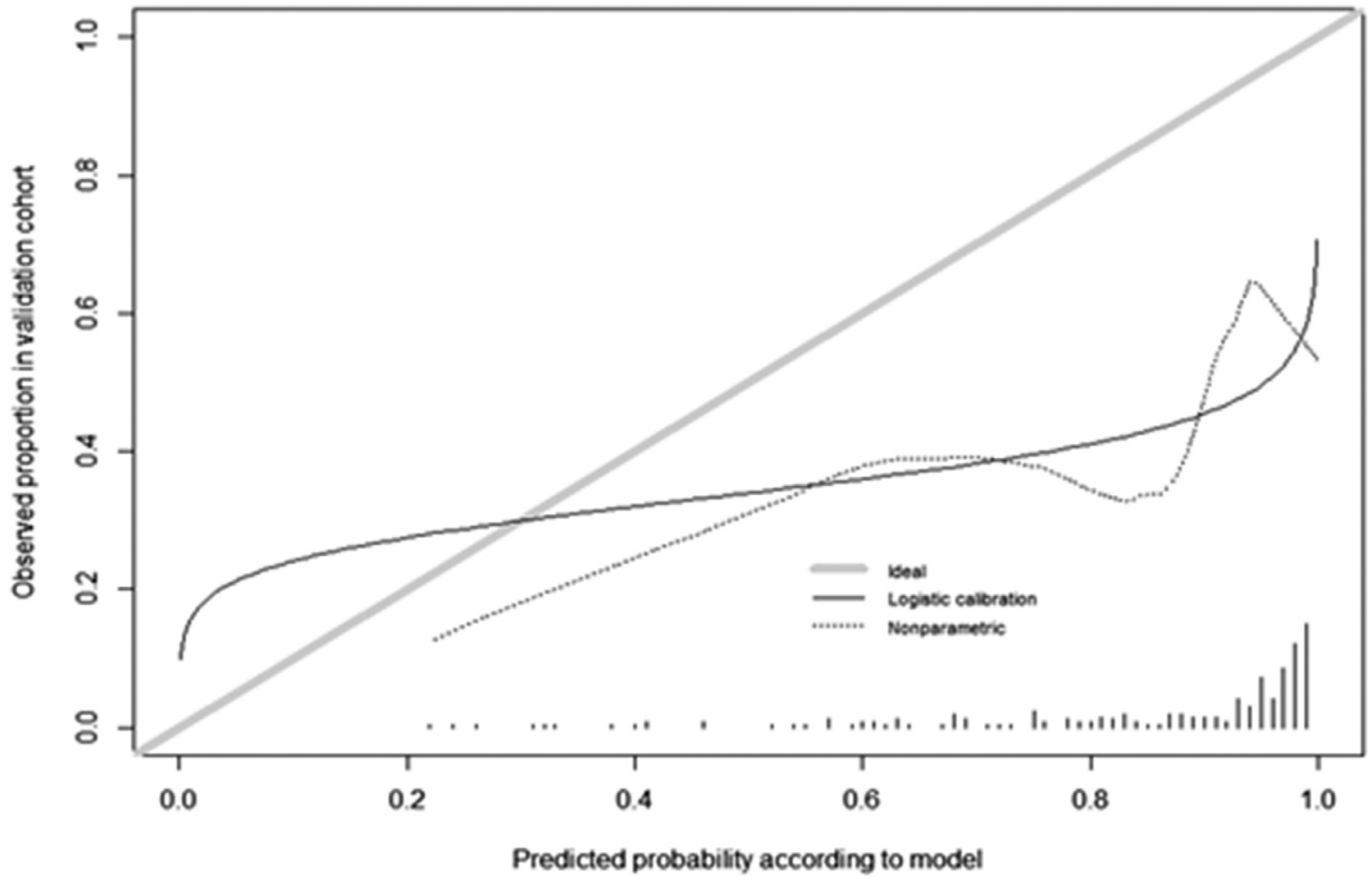

Figure 1. Calibration plot of external validation of previous original model in current study population, with the predictors age, current smoking, statin use, triglyceride, hypertension, history of cardiovascular diseases, body mass index (BMI), fasting plasma glucose.

Table 2. Possible predictors of persistent impaired glucose tolerance

\begin{tabular}{lcc}
\hline & aOR $(95 \% \mathrm{CI})$ & $95 \% \mathrm{CI}$ \\
\hline Age & 1.02 & $.99-1.05$ \\
Male & 1.42 & $.79-2.57$ \\
Current smoking & 1.61 & $.69-3.79$ \\
BMI & 1.08 & $.99-1.18$ \\
Hypertension & 1.70 & $.92-3.13$ \\
Statin use & 2.04 & $.96-4.33$ \\
Atrial fibrillation & 4.22 & $1.43-12.39$ \\
Previous ischemic & 0.70 & $.36-1.37$ \\
$\quad$ cardiovascular disease & & \\
Ischemic stroke vs TIA & .92 & $.52-1.64$ \\
Large artery atherosclerosis & 2.10 & $.91-4.84$ \\
$\quad$ vs other causes & & \\
Fasting glucose levels & 1.14 & $.66-1.95$ \\
$\quad$ per mmol/L & & \\
2-hour post-load glucose & 1.54 & $1.11-2.14$ \\
$\quad$ levels per mmol/L & & \\
HbAlc per mmol/mol & 1.11 & $1.00-1.23$ \\
\hline
\end{tabular}

Abbreviations: BMI, body mass index; HbA1c, glycolysated Haemoglobin 1c; TIA, transient ischemic attack.

Patients with persistent IGT might benefit from glucose-lowering therapy. With our prediction model, one could consider treating patients with the highest risk of developing persistent IGT in the acute phase after TIA or stroke. In a recent trial with nondiabetic patients with
Table 3. Predictors in the final model after correction for optimism

\begin{tabular}{lcr}
\hline & aOR & \multicolumn{1}{c}{$95 \% \mathrm{CI}$} \\
\hline BMI (per $\left.\mathrm{kg} / \mathrm{m}^{2}\right)$ & 1.05 & $.99-1.12$ \\
Hypertension & 1.48 & $.93-2.37$ \\
Statin use & 1.44 & $.81-2.56$ \\
Atrial fibrillation & 2.32 & $1.08-5.00$ \\
Large artery atherosclerosis & 1.43 & $.75-2.72$ \\
2-hour postload glucose levels & 1.08 & $1.01-1.16$ \\
Hbalc & 1.30 & $1.03-1.63$ \\
\hline
\end{tabular}

Formula prediction model: exp(linear predictor $) /(1+\exp [$ linear predictor]). Linear predictor $=-7.4706+.0529 \times \mathrm{BMI}+.3924 \times$ hypertension $+.3668 \times$ statin use $+.8444 \times$ atrial fibrillation $+.2598 \times 2$-hour postload glucose levels $+.0793 \times$ $\mathrm{Hbalc}+.3579 \times$ large artery atherosclerosis.

ischemic stroke or TIA, the risk of stroke or myocardial infarction was lower among patients who received pioglitazone in the acute phase than among patients who received placebo, and pioglitazone was also associated with a lower risk of diabetes. ${ }^{23}$

The Metformin and sitAgliptin in patients with IGT and a recent TIA or minor ischemic Stroke trial is recently completed and the results are expected soon, which is assessing the feasibility and safety of metformin and sitagliptin in patients with IGT and ischemic stroke or TIA. ${ }^{24}$

The strengths of our study are that it was prospectively designed and used easily accessible clinical variables in 


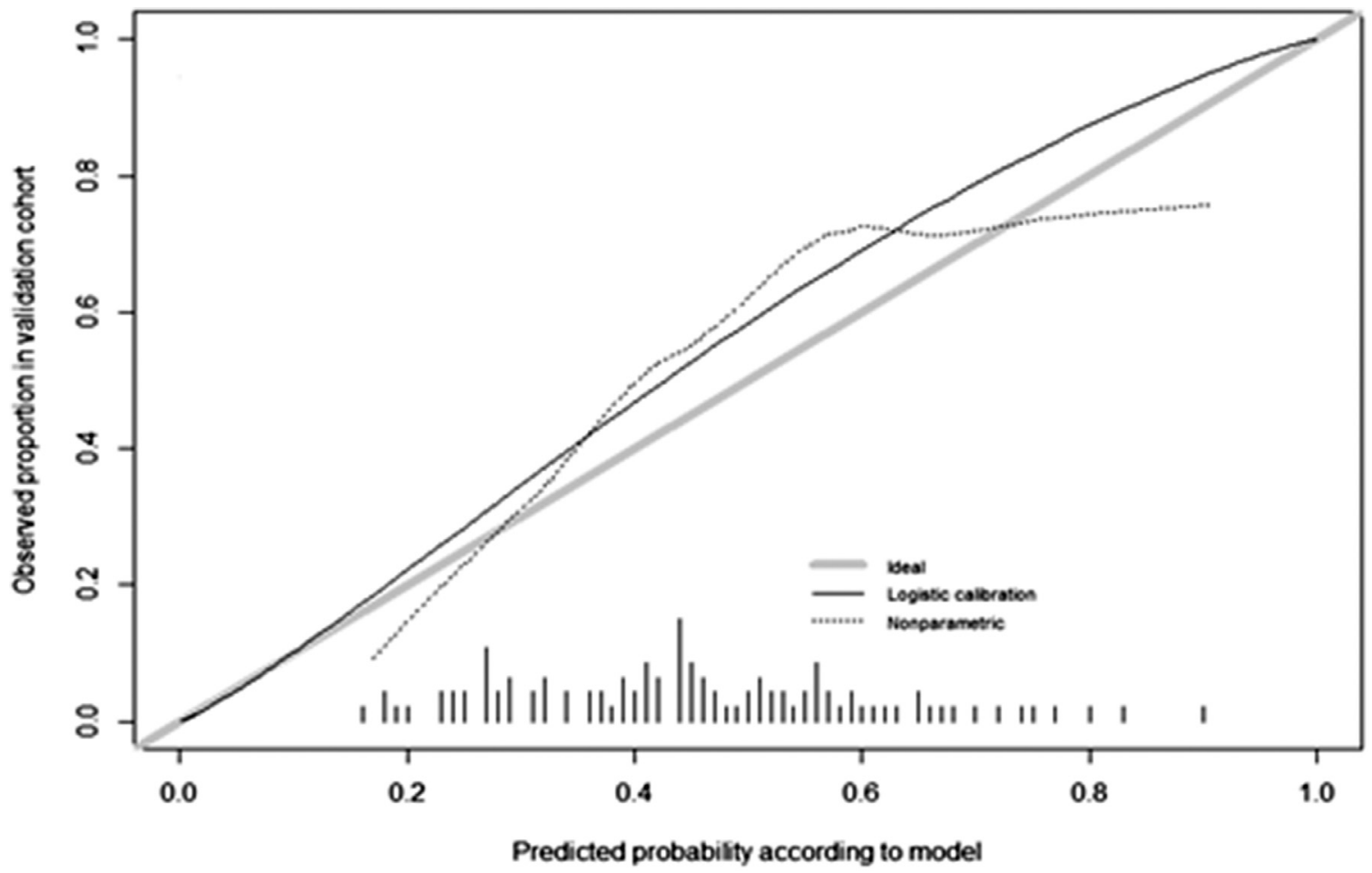

Figure 2. Calibration plot of external validation of newly developed prediction model in previous study population.

the prediction model. Also, we externally validated our prediction model, showing a good performance. However, we had missing variables which we tried to overcome with single imputation. Furthermore, we did not include patients with more severe ischemic strokes, so it is not certain whether the prediction model can also be used in this group of patients.

In conclusion, persistent IGT can accurately be predicted with our prediction model, using BMI, hypertension, statin use, atrial fibrillation, large artery atherosclerosis, 2-hour post-load glucose levels in the acute phase and $\mathrm{HbA1c}$ levels as clinical predictors. The model can be used to develop new strategies for secondary prevention in these patients.

\section{Conflict of Interest}

The authors state no conflicts of interests.

\section{References}

1. Dave JA, Engel ME, Freercks R, et al. Abnormal glucose metabolism in non-diabetic patients presenting with an acute stroke: Prospective study and systematic review. Qjm 2010;103:495-503.

2. Jia $Q$, Zheng $H$, Liu L, et al. Persistence and predictors of abnormal glucose metabolisms in patients after acute stroke. Neurol Res 2010;32:359-365.

3. Vancheri F, Curcio M, Burgio A, et al. Impaired glucose metabolism in patients with acute stroke and no previous diagnosis of diabetes mellitus. Qjm 2005;98:871-878.

4. Fonville S, den Hertog HM, Zandbergen AAM, et al. Occurrence and predictors of persistent impaired glucose tolerance after acute ischemic stroke or transient ischemic attack. J Stroke Cerebrovasc Dis 2014;23:1669-1675.

5. Vermeer SE, Sandee W, Algra A, et al. Impaired glucose tolerance increases stroke risk in nondiabetic patients with transient ischemic attack or minor ischemic stroke. Stroke 2006;37:1413-1417.

6. Lee M, Saver JL, Hong K-S, et al. Effect of pre-diabetes on future risk of stroke: meta-analysis. Bmj 2012;344:e3564.

7. Oizumi T, Daimon M, Jimbu Y, et al. Impaired glucose tolerance is a risk factor for stroke in a Japanese samplethe Funagata study. Metabolism 2008;57:333-338.

8. Jia $Q$, Liu G, Zheng $H$, et al. Impaired glucose regulation predicted 1-year mortality of Chinese patients with ischemic stroke: data from abnormal glucose regulation in Patients with acute stroke across China. Stroke 2014;45:1498-1500.

9. Adams HP, Bendixen BH, Kappelle LJ, et al. Classification of subtype of acute ischemic stroke. Definitions for use in a multicenter clinical trial. TOAST. Trial of Org 10172 in Acute Stroke Treatment. Stroke 1993;24:35-41.

10. American Diabetes Association. 2. Classification and Diagnosis of Diabetes: Standards of Medical Care in Diabetes-2020. Diabetes Care 2020;43:S14-S31.

11. Zou KH, O'Malley AJ, Mauri L. Receiver-operating characteristic analysis for evaluating diagnostic tests and predictive models. Circulation 2007;115:654-657.

12. Urabe T, Watada H, Okuma Y, et al. Prevalence of abnormal glucose metabolism and insulin resistance among subtypes of ischemic stroke in Japanese patients. Stroke 2009;40:1289-1295.

13. Perreault L, Temprosa M, Mather KJ, et al. Regression from prediabetes to normal glucose regulation is associated with reduction in cardiovascular risk: Results from the diabetes prevention program outcomes study. Diabetes Care 2014;37:2622-2631. 
14. Stefan N, Fritsche A, Schick F, et al. Phenotypes of prediabetes and stratification of cardiometabolic risk. Lancet Diabetes Endocrinol 2016;4:789-798.

15. Nichols GA, Hillier TA, Brown JB. Progression from newly acquired impaired fasting glusose to Type 2 diabetes. Diabetes Care 2007;30:228-233.

16. Nordestgaard BG, Varbo A. Triglycerides and cardiovascular disease. Lancet 2014;384:626-635.

17. Fonville S, Zandbergen AA, Vermeer SE, et al. Prevalence of prediabetes and newly diagnosed diabetes in patients with a transient ischemic attack or stroke. Cerebrovasc Dis 2013;36:283-289.

18. Haw JS, Galaviz KI, Straus AN, et al. Long-term sustainability of diabetes prevention approaches: A systematic review and meta-analysis of randomized clinical trials. JAMA Intern Med 2017;177:1808-1817.

19. Tabák AG, Herder C, Rathmann W, et al. Prediabetes: a high-risk state for diabetes development. Lancet 2012; 379:2279-2290.
20. The DECODE study group. Glucose tolerance and mortality: comparison of WHO and American Diabetic Association diagnostic criteria. Lancet 1999;354: 617-621.

21. Bartnik M, Malmberg K, Norhammar A, et al. Newly detected abnormal glucose tolerance: an important predictor of long-term outcome after myocardial infarction. Eur Heart J 2004;25:1990-1997.

22. Osei E, Fonville S, Zandbergen AAM, et al. Glucose in prediabetic and diabetic range and outcome after stroke. Acta Neurol Scand 2017;135:170-175.

23. Kernan WN, Viscoli CM, Furie KL, et al. Pioglitazone after Ischemic Stroke or Transient Ischemic Attack. N Engl J Med 2016;374:1321-1331.

24. Osei E, Fonville S, Zandbergen AAM, et al. Metformin and sitAgliptin in patients with impAired glucose tolerance and a recent TIA or minor ischemic Stroke (MAAS): study protocol for a randomized controlled trial. Trials 2015;16:1-6. 